Case report

\title{
Acupuncture might Increase Efficacy of Neoadjuvant Chemotherapy for Ultralow Rectal Cancer: A Case Report and Review of Related Literature
}

\author{
Xin Jiang1\#, Li-na Wang2\#, Qiao-li Zhang2,Ye-hong Tian²,Ming Yang 1,Yu-xiang Wan 2 ${ }^{2}$ Xue-wei Qi², Li Yao³, Jin-chang \\ Huang $^{*}$ \\ 1School of Traditional Chinese Medicine, Beijing University of Chinese Medicine, Beijing, China \\ 2The Third Affiliated Hospital, Beijing University of Chinese Medicine, Beijing, China \\ ${ }^{3}$ Fourth Department of General Surgery, China-Japan Friendship Hospital, Beijing, China
}

Received March 28, 2019; Accepted April 10, 2019

\begin{abstract}
Background: We report a case of a patient with ultra-low rectal cancer who had a successful anus-preserving operation with fire needling to increase the efficacy of neoadjuvant chemotherapy and an overview of the related literature.

Case presentation: A 38-year-old male with a diagnosis of rectal cancer (cT4N0-2M0 Stage III), the lower edge of the tumor was $2 \mathrm{~cm}$ away from the anal margin. He had a strong desire for anal preservation and received six cycles of neoadjuvant chemotherapy by FOLFOX6 regimen at the China-Japan Friendship Hospital. The tumor shrinkage was not significant, and then fire-needling acupuncture was added to the original plan at baliao acupoints during the period of chemotherapy. After another six cycles of acupuncture, the tumor disappeared in the imaging examination. He underwent general anesthesia for laparoscopic rectal cancer (ISR) radical surgery.
\end{abstract}

Results: The specimen showed no tumor, and no metastatic carcinoma was found in the peri-intestinal lymph nodes.

Conclusion: Acupuncture combined with chemotherapy may increase the anti-tumor effect, which could be of great significance for ultra-low rectal cancer patients if evidence can be replicated in additional study. Further case series research is needed.

\section{KEYWORDS}

Fire needling acupuncture; ultralow rectal cancer; neoadjuvant chemotherapy; case report

Colorectal cancer is one of the most common malignant tumors around the world, and low, ultra-low rectal cancer accounts for $70-80 \%$ of all rectal cancers in China(1). The treatment of low rectal cancer emphasizes surgery-based multidisciplinary treatment including radiotherapy and chemotherapy(2). According to Miles who first described the operation of abdominoperineal resection (APR)(3), there was no possibility of preserving anal sphincter in low and ultra-low rectal cancer (4). However, more and more studies have found that the tumor infiltrates into the distal intestinal wall rarely exceeds $2 \mathrm{~cm}$, and good oncological prognosis can be guaranteed when the distal resection distance is more than $2 \mathrm{~cm}$ (5). The emergence of the $2 \mathrm{~cm}$ rule dramatically expands the anus sphincter-preserving scope of low and ultra-low rectal cancer. In this article, we reported a case that ultra-low rectal cancer $2 \mathrm{~cm}$ away from the anal margin was resected, and the anus was pre-

\footnotetext{
\# Xin Jiang and Li-na Wang contributed equally to this work.

${ }^{*}$ Correspondence to Prof. Huang Jinchang, e-mail: zryhhuang@163.com.
}

served by fire needling acupuncture combined with chemotherapy.

\section{CASE PRESENTATION}

A 38-year-old man with recurrent bloody stool, paroxysmal abdominal pain, and difficulty in defecation was referred to the China-Japan Friendship Hospital for urgent preservation of anal function. On October 17, 2016, enteroscopy showed circumferential protuberant lesions $2-4 \mathrm{~cm}$ away from the anus with a rough surface and bleeding upon touch. The lower rectal wall was irregularly thickened. The lower margin of the lesion was about $2 \mathrm{~cm}$ away from the anal margin. Pelvic contrast-enhanced magnetic resonance imaging (MRI) showed the length of the lesion was about 5-6 cm, the intrinsic muscle layer of the lesion was rough, and the boundary between the lesion and the right levator ani muscle was not clear. Several swollen lymph nodes were seen in the peripheral space between the bilateral pelvic wall and the straight intestine; the 
larger was about $0.7 \mathrm{~cm}$ (Figure 1). High-grade intraepithelial neoplasia (rectum) was considered in puncture pathology, malignancy is not excluded. After a multidiscciplinary team consultation, the diagnosis was rectal mali- gnancy (cT4N0-2M0 Stage III), considering that the tumor was large, invading the surrounding tissues and swollen lymph nodes exceeded $0.5 \mathrm{~cm}$.

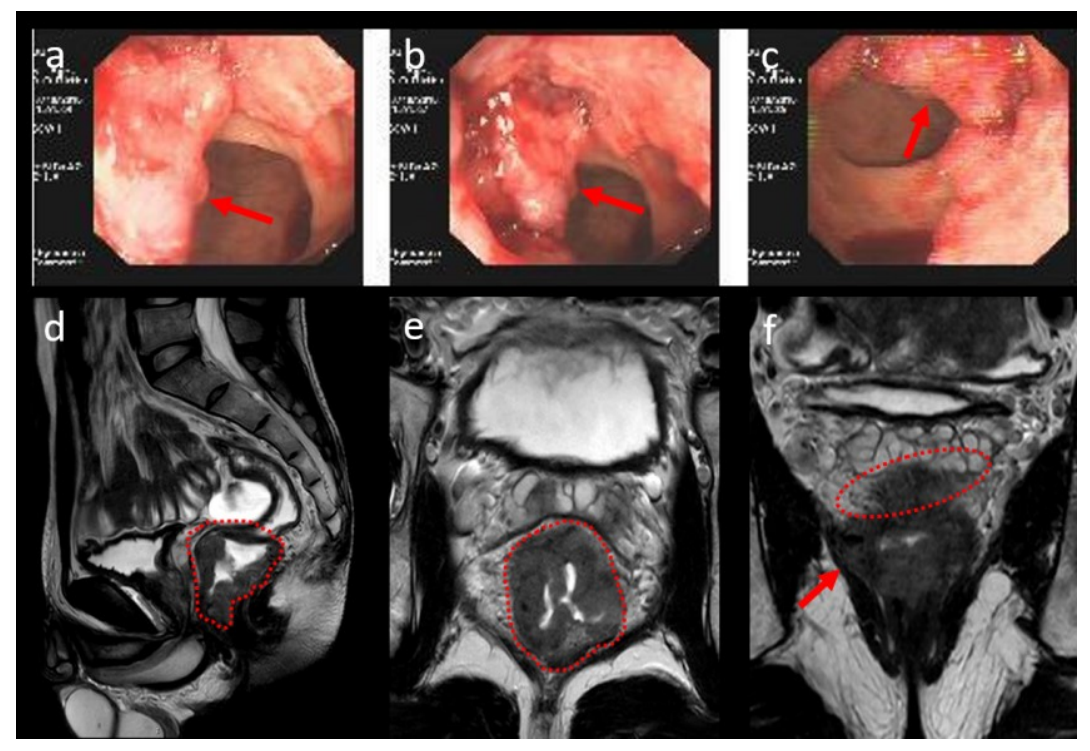

Figure 1. Colonoscopy and MRI examination before chemotherapy. $a, b$ and $c$ were tumor uplift under the colonoscopy field of view, with a rough surface and bleeding; $d$ and e were MRI findings in coronal and transverse positions; f. The boundary between the tumor and the levator ani muscle on the right was unclear, and the lesion edge was rough at the intrinsic muscle level.

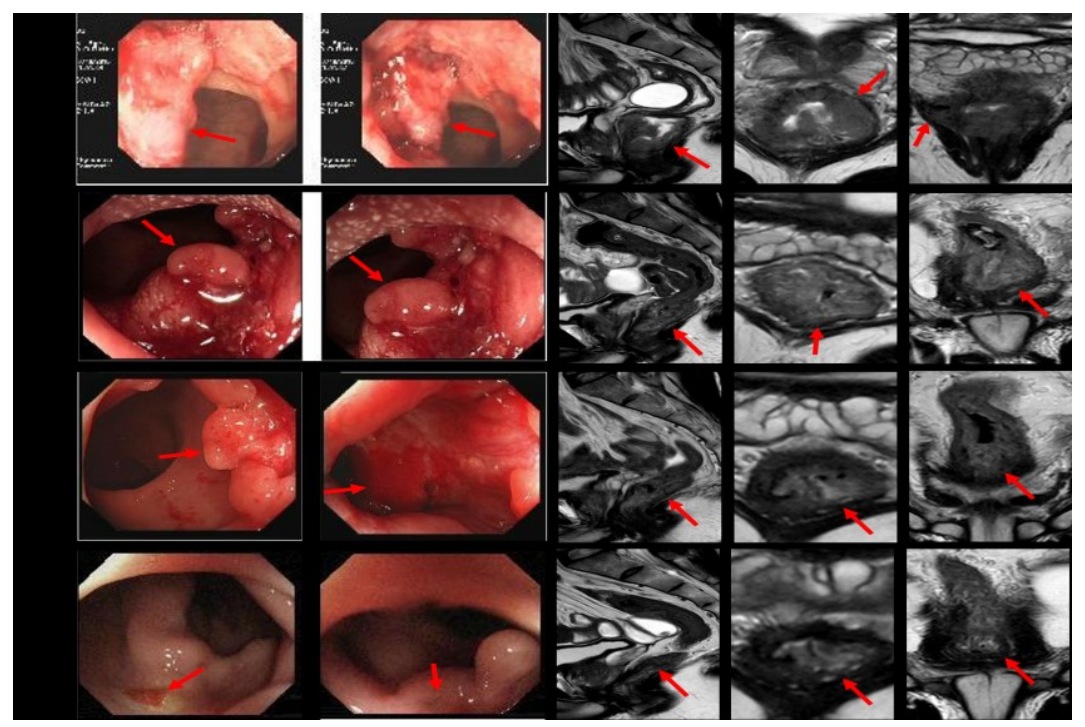

Figure 2. Tumor changes during treatment by colonoscopy and MRI examination

According to the National Comprehensive Cancer Network (NCCN) guidelines (2015 edition), preoperative concurrent chemoradiotherapy is recommended. Considering there are defecation disorders, urinary incontinence, and intestinal dysfunction after preoperative radiotherapy, and there is a risk of anastomotic fistula $(6,7)$, FOLOX6 regimen was used for 6 cycles (October 2016 to January
2017). The tumor shrinkage was not evident, but watery stools, fever, nausea, vomiting, and hand-foot syndrome occurred. Subsequently, after a multidisciplinary consultation decision, the fire needling acupuncture on Baliao, Yaoyangguan, and Changqiang was given on the first, third, and fifth days of the original chemotherapy regimen for another six cycles (February-May 2017). Imaging examin- 
ation showed that the tumor disappeared after acupuncture was completed (Figure 2).

The patient underwent laparoscopic intersphincteric resection (ISR) and ileostomy under general anesthesia on June 21, 2017, and the tumor was removed entirely. Postoperative pathology: No tumor tissue was found in the samples of the specimen, with fibrous tissue hyperplasia and scattered calcification. The upper and lower ends and the circumferential margin were clean, and no cancer metastasis was found in the peri-intestinal lymph nodes $(0 / 19)$ (Figure 3$)$. The patient received ostomy return surgery on July 31,2018 , and the patient retains some of the anal function, although most of the anal sphincter was resected.
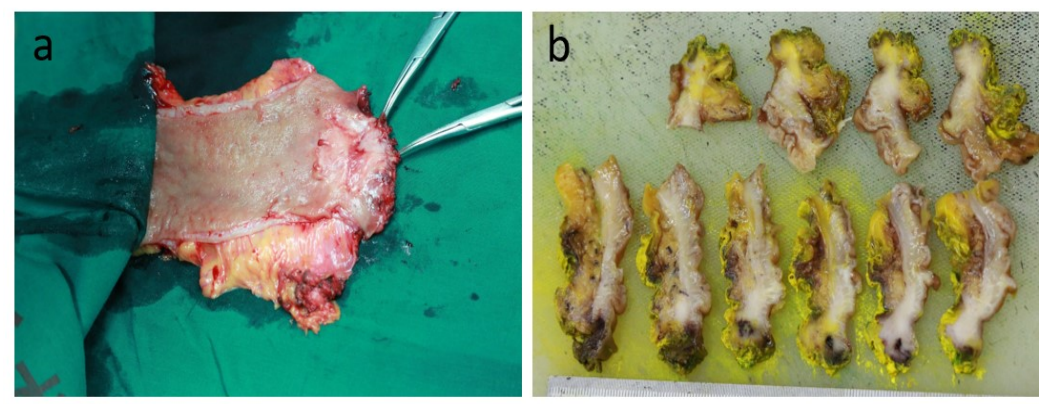

C

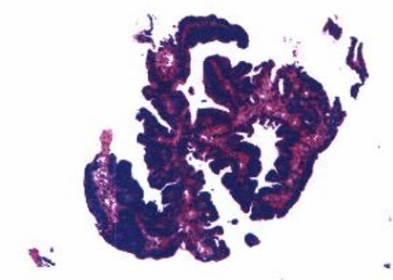

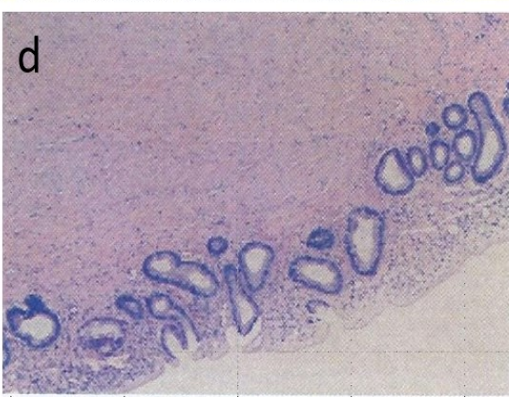

Figure 3.Gross specimen and staining. a. intraoperative gross specimen; b. Fixed section of specimens; c. HE staining of preoperative specimens; d postoperative specimen HE staining

\section{DISCUSSION}

Surgery is still the most important radical treatment for rectal cancer, and the most important reference factor for performing anal sphincter preservation surgery for lower rectal cancer is the distance from the lower edge of the tumor to the anal margin. Anatomical studies have shown that the anal margin is $2.5 \mathrm{~cm}$ from the dentate line, and 0.5 $\mathrm{cm}$ above the dentate line is the upper edge of the levator ani muscle. Therefore, to ensure the integrity of levator ani muscle, at least $3 \mathrm{~cm}$ of anorectum should be preserved after resection of tumors, and $2-3 \mathrm{~cm}$ of the normal intestinal tube should be resected at the lower edge of the tumors. The distance between the lower edge of the tumors and the anal edge should be at least $5-6 \mathrm{~cm}$ before anus-preserving surgery can be performed. Recent literature indicates that distal resection distances $<2 \mathrm{~cm}$ and $>2$ $\mathrm{cm}$ did not affect oncologic prognosis with intraoperative frozen sections, and even a $5 \mathrm{~mm}$ distal margin was safe (8).

Anal function preservation is an urgent desire for patients with low rectal cancer. How to improve the anus presservation rate of middle and low rectal cancer and to reduce the local recurrence and the incidence of anastomotic leakage after an operation is still a major clinical problem. This goal leads to the use of preoperative neo-adjuvant chemotherapy(9). Neoadjuvant chemotherapy can reduce the size of the tumors, increase the distance between the lower edge of the tumors and the anus, and create favorable conditions for radical operation of rectal cancer. However, chemotherapy resistance may not only weaken the efficacy of chemotherapy but also lead to the failure of chemotherapy. The patient reported was with a more massive rectal tumor, a lower tumor edge which was only $2 \mathrm{~cm}$ away from the anal margin, a unclear tumor boundary near the levator ani muscle on the right side, accompanied by lymph node metastasis, and a strong desire for anus preservation, the efficacy of neoadjuvant chemotherapy directly determines the success of anus preservation.

Improving the efficacy of chemotherapy has always been the main aim in oncology. Increasing the local drug concentration of intravenous chemotherapy is of great significance to improve the efficacy of chemotherapy. Investigators continue to explore targeted delivery systems such as intratumoral injection (10), interventional chemotherapy (11), and drug-loaded particles(12). In the ancient literature of Chinese medicine, there are records of fire needling therapy for tumors, but fire-needling therapy for tumors has rarely been used in modern times. In this report, we administered the fire needling acupuncture the- 
rapy concurrently with chemotherapy in one patient, and the tumor disappeared completely. Acupoints such as Baqiao, Yaoyangguan, and Changqiang are close to the location of low rectal cancer; and acupuncture at these points can exert specific stimulation in the local area, appeared to significantly increase the efficacy of chemotherapy.

In our mouse model research of acupuncture enhancement of anticancer effect of chemotherapy drugs (13), we found that the inhibition rate, defined by the percentage of tumor shrinkage, in peritumoral acupuncture combined with cyclophosphamide $(53.68 \%)$ was significantly $(\mathrm{p}<0.05)$ better than that of cyclophosphamide $(31.62 \%)$ or acupuncture alone $(34.56 \%)$. This suggests that acupuncture and chemotherapy interactively inhibit the growth of $4 \mathrm{~T} 1$ breast cancer in mice. One possible explanation is that the process of angiogenesis in local tumors may be modified. Another intriguing possibility is that peritumoral acupuncture may increase the drug concentration of tumors by promoting the normalization of blood vessels and thus increasing the efficacy of chemotherapy. These observa-tions warrant a further investigation in a larger patient sample and the potential mechanisms by which acupuncture, chemotherapy, and their interaction may enhance the anticancer effect on solid tumors.

\section{CONFLICTS OF INTEREST}

The authors declare that there is no conflict of interest regarding the publication of this paper.

\section{ETHICAL APROVAL}

The study was examined and approved by the institutional review board of the third hospital affiliated to Beijing University of Chinese Medicine (Approval No. BZYSY-YJKT-3.1). Written informed consent was obtained from the patients for the information to be included in our manuscript. His information has been de-identified to the best of our ability to protect his privacy.

\section{FUNIDNG}

Supported by the National Natural Science Foundation for Young Scientists of China (No. 81804177) and Beijing Municipal Natural Science Foundation (No.7172128).

\section{REFERENCES}

1. Mao J, Wu P, Yang G, Wu P. Clinical efficacy of anus-preserving operation for ultra low rectal cancer. Chin J Dig Surg. 2015; 14(6):461-5.

2. Aggarwal B, Prasad S, Sung B, Krishnan S, Guha S. Prevention and Treatment of Colorectal Cancer by Natural Agents From Mother Nature. Curr Colorectal Cancer Rep. 2013;9(1):37-56.

3. Corman ML. Classic articles in colonic and rectal surgery. A method of performing abdominoperineal excision for carcinoma of the rectum and of the terminal portion of the pelvic colon: by W. Ernest Miles, 1869-1947. Dis Colon Rectum. 1980;23(3):202-5.

4. Marks J, Nassif G, Schoonyoung H, DeNittis A, Zeger E, Mohiuddin $\mathrm{M}$, et al. Sphincter-sparing surgery for adenocarcinoma of the distal $3 \mathrm{~cm}$ of the true rectum: results after neoadjuvant therapy and minimally invasive radical surgery or local excision. Surg Endosc. 2013;27(12):4469-77.

5. Zuo X, Meng X. Recent advances in the safe distance of the distal excisionmargin for rectal cancer. International Journal of Surgery. 2011;38(8):548-52.

6. Contin P, Kulu Y, Bruckner T, Sturm M, Welsch T, Muller-Stich $\mathrm{BP}$, et al. Comparative analysis of late functional outcome following preoperative radiation therapy or chemoradiotherapy and surgery or surgery alone in rectal cancer. Int J Colorectal Dis. 2014;29(2):165-75.

7. Lange MM, den Dulk M, Bossema ER, Maas CP, Peeters KC, Rutten $\mathrm{HJ}$, et al. Risk factors for faecal incontinence after rectal cancer treatment. Br J Surg. 2007;94(10):1278-84.

8. Bujko K, Rutkowski A, Chang GJ, Michalski W, Chmielik E, Kusnierz J. Is the $1-\mathrm{cm}$ rule of distal bowel resection margin in rectal cancer based on clinical evidence? A systematic review. Ann Surg Oncol. 2012;19(3):801-8.

9. Frei E, 3rd. Clinical cancer research: an embattled species. Cancer. 1982;50(10):1979-92.

10. Liu Z, Ballinger JR, Rauth AM, Bendayan R, Wu XY. Delivery of an anticancer drug and a chemosensitizer to murine breast sarcoma by intratumoral injection of sulfopropyl dextran microspheres. J Pharm Pharmacol. 2003;55(8):1063-73.

11. Wang SG, Mu N, Sun HY. Effect of Interventional Therapy on the Expression of Survivin mRNA in Cervical Cancer. Anticancer Res. 2017;37(8):4707-10.

12. Masood F. Polymeric nanoparticles for targeted drug delivery system for cancer therapy. Materials Science and Engineering: C. 2016;60(1):569-78.

13. Tian Y, Jiang X, Zhao W, Pan L, Li B, Wang Z, et al. Acupuncture enhances anticancer effects of cyclophosphamide on $4 \mathrm{~T} 1 \mathrm{tu}-$ mors via suppression of angiogenesis in BALB/c mice. Journal of Traditional Chinese Medical Sciences. 2017; 4(2): 21621.

How to cite this article:

Jiang X, Wang L, Zhang Q, Tian Y, Yang M, Wan Y, Qi X, Yao L, Huang J. Acupuncture might increase efficacy of neoadjuvant chemotherapy for ultralow rectal cancer: a case report and review of related literature. Glob Clin Transl Res. 2019; 1 (2):74-77. 